\title{
De fronteras y enClaVes: LA PRESENCIA NASCA EN LA SIERRA De ICA (260 A.C. -640 D.C.)
}

\author{
Kevin Lane ${ }^{a}$, Oliver Huaman ${ }^{b}$, Luis Coll ${ }^{c}$, Alexander Pullen ${ }^{d}$, \\ David Beresford-Jones ${ }^{e} y$ Charles French ${ }^{f}$
}

\begin{abstract}
Resumen
Las investigaciones iniciadas en el año 2014 en el Cerro San Bernardo (ACO3; 2000 metros sobre el nivel del mar) y Challaca (CH1; 2015 metros sobre el nivel del mar) nos han permitido hallar los primeros sitios Nasca (260 a.C a 640 d.C.) en la ecozona yunga de la cuenca alta del rio Ica. Los dos sitios también registran la posibilidad de una ocupación aún más temprana que se remonta al Horizonte Temprano (840 a 260 a.C.). Estos dos sitios se ubican sobre cerros pequeños, que están conformados principalmente por granito, y que se localizan al norte y sur sobre los márgenes de la cuenca alta del rio Ica. Desde estos sitios, se puede divisar una extensa área de cultivo que se extiende desde los cerros al este, hasta el oeste y el límite del cañón de Challaca-Tiraxi, la cual podría estar bajo su control. Geográficamente, el cañón divide la sierra de Ica del área costera, a partir de lo cual le brinda una ubicación geopolitica importante. Es posible que la localización estratégica de este enclave Nasca en la sierra de Ica haya sido para controlar el acceso desde las sociedades costeras a los sectores de la sierra, mientras se nutría de los recursos de esta rica zona alta. Probablemente, la ocupación de este punto estratégico se inició durante el Horizonte Temprano (840 a 260 a.C.), lo cual significaría una presencia larga por parte de los grupos costeños en la sierra. Ante este contexto, el objetivo del presente trabajo es indagar sobre los patrones de control, interacción y el tipo de frontera entre los Nasca y los grupos culturales serranos, vecinos, sincrónicos, aportando nuevas evidencias sobre la presencia Nasca en la cuenca alta del rio Ica.
\end{abstract}

Palabras clave: sierra de Ica, Nasca, Horizonte Temprano, Periodo Intermedio Temprano, frontera, enclave

\section{Abstract}

OF ENCLAVES AND FRONTIERS: THE NASCA PRESENCE IN THE ICA HIGHLANDS (260 B.C. - A.D. 640)

During 2014, research undertaken at the sites of Cerro San Bernardo (ACO3; 2,000 m above sea level) and Challaca (CH1; 2,015 m above sea level) revealed the first documented Nasca sites (260 B.C-A.D. 640) in the yunga ecozone of the upper drainage of the Ica River. Both sites also demonstrated evidence of an even earlier, Early Horizon occupation $(840-260$ B.C.). These two sites are located on small, mainly granite, knolls situated on the Northern and

${ }^{a}$ CONICET - Instituto de Arqueología, FFyL, Universidad de Buenos Aires

Correo electrónico: kevin.lane@cantab.net

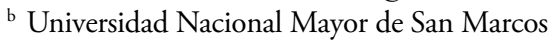

Correo electrónico: oliverhuaman@gmail.com

c Universidad de Buenos Aires, FFyL, IDECU (Instituto de las Culturas - UBA / CONICET)

Correo electrónico: luisvjcoll@hotmail.com

${ }^{d}$ University of Cambridge, McDonald Institute for Archaeological Research

Correo electrónico: agp27@hotmail.com

${ }^{\mathrm{e}}$ University of Cambridge, McDonald Institute for Archaeological Research

Correo electrónico: dgb27@cam.ac.uk

${ }^{f}$ University of Cambridge, McDonald Institute for Archaeological Research

Correo electrónico: caif2@cam.ac.uk 
Southern banks of the upper drainage of the Ica River. From these sites, it is possible to oversee an extensive area of cultivation that extends from the mountains to the East down to the Western boundary of the Challaca-Tiraxi Canyon. An area, which these sites could well have controlled. Geographically, the canyon divides the Ica Highlands from the coastal area, giving this juncture geopolitical importance. It is possible that the strategic location of this Nasca enclave in the Ica Highlands was aimed at controlling access between coastal polities and highland sectors, while exploiting this zones rich, high-altitude resources. Probably, occupation of this strategic sector commenced during the Early Horizon (840260 B.C.), pointing to a long presence by coastal groups in the highlands. The aim of this article then, is to tease-out patterns of control, interaction, and the type of frontier in existence between the Nasca and coeval, neighboring, highland cultural groups, thereby providing new evidence concerning Nasca presence in the upper drainage of the Ica River.

Keywords: Ica Highlands, Nasca, Early Horizon, Early Intermediate Period, frontier, enclave

\section{Introducción}

Tradicionalmente, los estudios sobre la extensión geográfica de la cultura Nasca se han centrado en la costa sur-central (Silverman y Proulx 2002), aunque se ha propuesto que su influencia cultural se extendió tanto hacia el norte y el sur de la costa como hacia el este hasta las tierras altas de Ayacucho (Proulx 2008: 564). A pesar de las actuales interpretaciones acertadas en el énfasis de la naturaleza costero y ribereńa de la cultura política Nasca (Beresford-Jones 2011), nuevas investigaciones han dado luz sobre la penetración nasca en las áreas de yunga y kichwa en las tierras altas en el valle Palpa (Reindel 2012; Reindel et al. 2013; Reindel et al. 2015). Estudios recientes en las tierras altas de Ica están demostrando un similar patrón de ocupación nasca, al menos, en la ecozona yunga, que, posiblemente, generan un desplazamiento o absorción de un asentamiento temprano Paracas anterior. En este artículo, describimos la presencia Nasca en las tierras altas de Ica, destacando la naturaleza física de dicha presencia y la posible función detrás de su existencia (Fig. 1). Precisamente, nuestro objetivo es indagar sobre los patrones de control, interacción y la naturaleza de la frontera entre los Nasca y los grupos culturales colindantes.

\section{One River Project (ORP) 2012-2017}

Este estudio forma parte del One River Project (French et al. 2012), cuyo objetivo es realizar un seguimiento sobre el cambio a través del tiempo en el florecimiento y colapso de la riqueza cultural a lo largo de un río — río Ica- desde su nacimiento en los Andes sur-centrales hasta su desembocadura en la costa del desierto. La región andina es uno de los corazones excepcionales de la humanidad tanto para la agricultura como para el estudio de la civilización "prístina». Su costa pacífica tiene uno de los desiertos más secos del mundo, cuyos recursos de agua son únicamente los ríos alimentados por las precipitaciones que se generan en el interior de las tierras altas. Los Andes, después del Himalaya, se constituyen como la segunda área mundial en incluir una enorme variación ambiental para la ecología humana (Shimada 1985). Además, por su ubicación en estas latitudes tropicales, permiten el desarrollo de cultivos, incluso, en altitudes extremas por encima de los 4000 metros sobre el nivel del mar.

A pesar de la obvia interdependencia entre la hidrología y la economía de la costa y sierra, aún son pocas las investigaciones que han abordado este tema con este tipo de enfoque de análisis, que abarca por completo el curso de un río dentro de una sola cuenca, pese a que es esta la escala de análisis más apropiada para los estudios sobre los modelos referentes a los cambios de asentamientos, el uso de tierra, el manejo de agua y al desarrollo de culturas. En los últimos cuatro ańos, hemos llevado a cabo una arqueología del río Ica desde una perspectiva temporal prolongada que abarca el Período Precerámico Medio (c. 8000-4500 AP) hasta los primeros tiempos del Imperio español (1532-1615 d.C.) y bajo esta escala de análisis espacial amplia. 


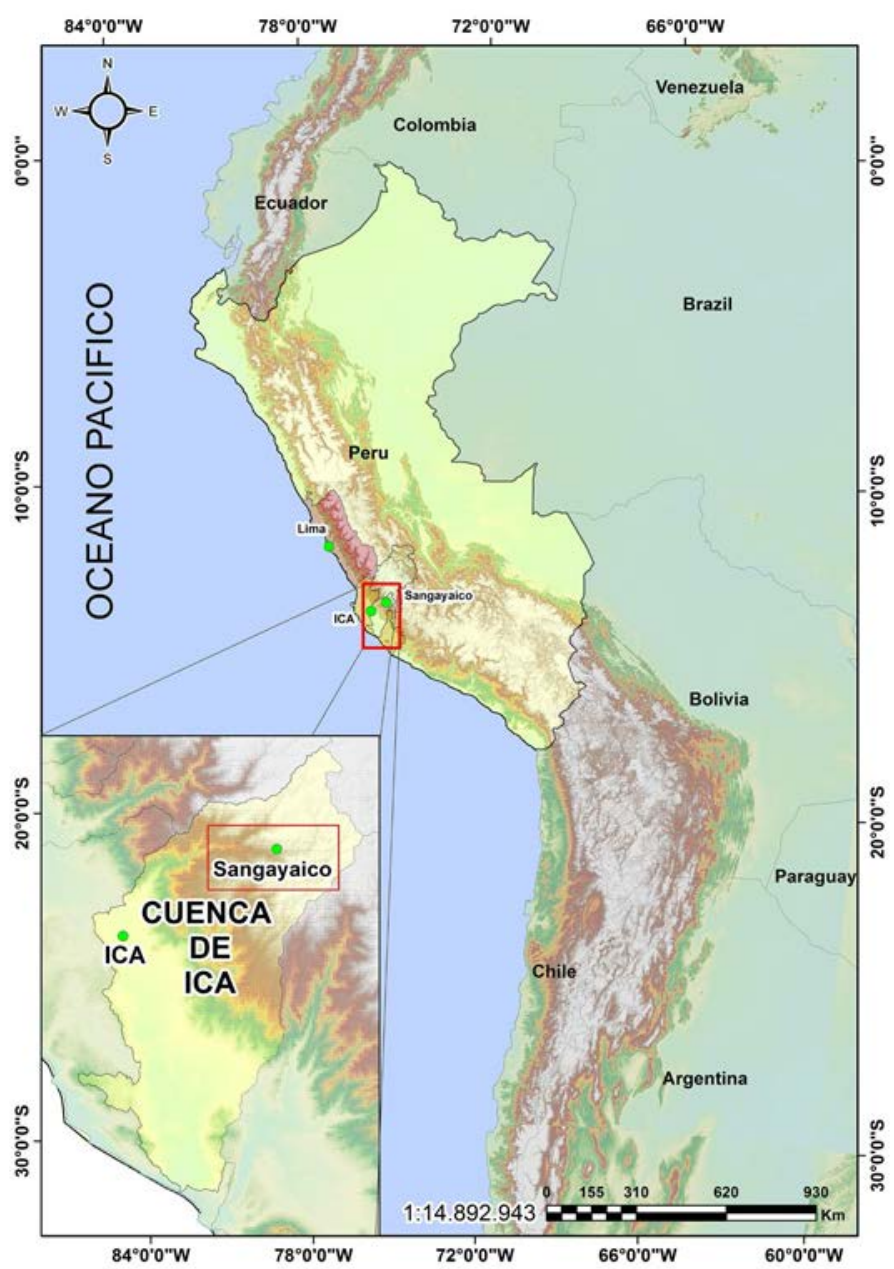

Figura 1. Mapa del área de estudio (One River Project - Proyecto de Investigación Arqueológica Cuenca de Ica ORP-PIACI 2014).

Si bien la cuenca baja del valle Ica ha sido, al menos, parcialmente estudiada (v.g. Engel 1957; Cook 1991; Massey 1991; Beresford-Jones 2011; Bachir Bacha y Llanos 2013; Beresford-Jones et al. 2016), la cuenca alta nunca ha sido objeto de investigaciones arqueológicas previas. En ese sentido, nuestro estudio ha sido uno de los primeros en esta región y, hasta el momento, ha permitido relevar 56 sitios nuevos a lo largo de dos ríos tributarios laterales al río Ica: los ríos Olaya y Santiago. Esto nos ha permitido generar una plena confianza en que futuros trabajos de campo en otros afluentes revelarán aún más sitios. De los 56 sitios registrados hasta el momento, solo tres muestran evidencias indiscutibles de ocupación con cronologías pertenecientes previas al Período Intermedio Tardío o al primer período de la Colonia española (1000-1615 d.C.).

La razón de esta insuficiencia de datos sobre sitios más tempranos es atribuibles diversos factores que van desde la conservación preeminente de los sitios tardíos las destrucciones o sustituciones de estos a causas naturales o por la acción humana. Incluso, existe hasta un posible sesgo de análisis que genera que los sitios más deslumbrantes visualmente y cronológicamente tardíos se descubran y registren en detrimento de los sitios con cronologías más tempranas y características más efímeras.

De los tres sitios con ocupaciones tempranas, dos-CH1 y ACO3- son objeto de estudio en el presente artículo, mientras que el tercero - Huinchocruz (SAN4) - es un posible sitio ritual que se ubica sobre una imponente cima localizada sobre el río Olaya en los sectores bajos de la cuenca 
de Ica a 4070 metros sobre el nivel del mar. Este sitio está conformado por tres componentes culturales principales: una plataforma subcircular o ushnu; un complejo huanca, compuesto por una huanca ubicada dentro de una estructura de pirca circular; y un calvario cristiano. Tanto la cerámica como los restos materiales arqueológicos del sitio SAN4 indican que el sitio fue usado desde el Período del Horizonte Temprano (840-260 a.C.) hasta el presente. Es probable que haya representado la ubicación de una importante huaca local prehispánica, antes de la subsecuente evangelización cristiana y su transformación como el centro de adoración durante la Fiesta de las Cruces celebrada durante el mes de mayo.

\section{Trabajo de campo en Challaca (CH1) y Cerro San Bernardo (ACO3)}

En relación con los dos sitios considerados en el presente artículo (Fig. 2), el trabajo de campo fue realizado durante el año 2014 en Challaca (CH1) y Cerro San Bernardo (ACO3) después de sus descubrimientos iniciales en 2013 (Huamán Oros y Lane 2014; Chauca Iparraguirre y Lane 2015). Esto conllevó al desarrollo principalmente del registro de cada uno y a una serie de sondeos en $\mathrm{CH} 1$ junto a una recolección preliminar de material de superficie en ACO3.

Ambos sitios están localizados cerca uno del otro, en lomas de granito opuestas que se ubican a las márgenes del río Ica. Ecológicamente, los sitios se encuentran en la parte baja de las tierras altas, que es conocida como yunga. ACO3 está situado al oeste con una altitud de aproximadamente 2000 metros sobre el nivel del mar, mientras que $\mathrm{CH} 1$ se encuentra a cinco kilómetros aguas arriba hacia el este con una altitud de alrededor de 2015 metros sobre el nivel del mar. Además, ambos se encuentran ubicados a lo largo del borde de un área rica en tierra agrícola que es conformada por el cañón Challaca-Tiraxi (1200 a 1800 metros sobre el nivel del mar), el cual divide a esta región de la yunga ribereńa del valle costero Ica.

La localización del cañón Challaca-Tiraxi - línea divisoria entre la cuenca baja y la cuenca media del valle Ica - forma un cuello de botella de sedimentos que genera un área muy productiva con ricos nutrientes, la cual es alimentada por las aguas de los ríos Tambo y Ica. Debido a que el cañón Challaca-Tiraxi atrapa eficazmente limos hacia el este, esto a su vez genera un área de producción enriquecida que, junto a las condiciones más benignas de la yunga — la temperatura, la altitud, la radiación solar - en gran medida, aumenta el potencial económico de esta tierra. La ubicación del $\mathrm{CH} 1$ y ACO3 en las colinas rocosas maximiza la disponibilidad de estas tierras productivas; estas se ubican estratégicamente, puesto que el estar en las alturas les permite tener un control importante. El posicionamiento de los importantes centros tardíos del Período Intermedio Tardío (1000-1450 d.C., v.g. ACO1-2, ACO4-5, CH10-13) cercano a estos dos sitios (CH1 y ACO3) avala la importancia de la continuidad de esta zona productiva durante los períodos posteriores. Cabe la posibilidad de que estos sitios del Intermedio Tardío estuvieran físicamente sobre sitios más tempranos, aunque hasta la fecha no hemos encontrado alguna indicación de esto. De hecho, hasta el presente, esta área es la más productiva a nivel agrícola de esta región de la sierra.

Las características internas de ambos sitios son muy similares, a pesar de que el sitio ACO3 está mucho mejor preservado que el sitio $\mathrm{CH} 1$, por lo que el primero (ACO3) sirve como patrón básico para estos dos sitios. Este patrón básico es una estructura circular rodeada por una serie de recintos más pequeños en forma de «D», que componen una disposición similar a formas de pétalos. Tanto $\mathrm{CH} 1$ como ACO3 tienen evidencias de al menos dos de estos complejos circulares y recintos en «D», con diámetros que rondan entre los 10 y 15 metros. Estas estructuras son semejantes a las registrados por el equipo de Marcus Reindel en el alto valle de Palpa y con cronologías que se ubican en el Horizonte Temprano y en el Período Intermedio Temprano (Reindel 2012; Reindel et al. 2013; Reindel et al. 2015; Sossna 2016). Sin embargo, cabe señalar que los dos sitios del valle de Ica son más pequeños e, incluso, el complejo "círculo y pétalos» es también más pequeño que su análogo en el valle de Palpa.

También, registramos, tanto en Challaca como en el Cerro San Bernardo, una serie de terrazas de poca profundidad construidas en dirección a la llanura de inundación del río. En CH1, estas 


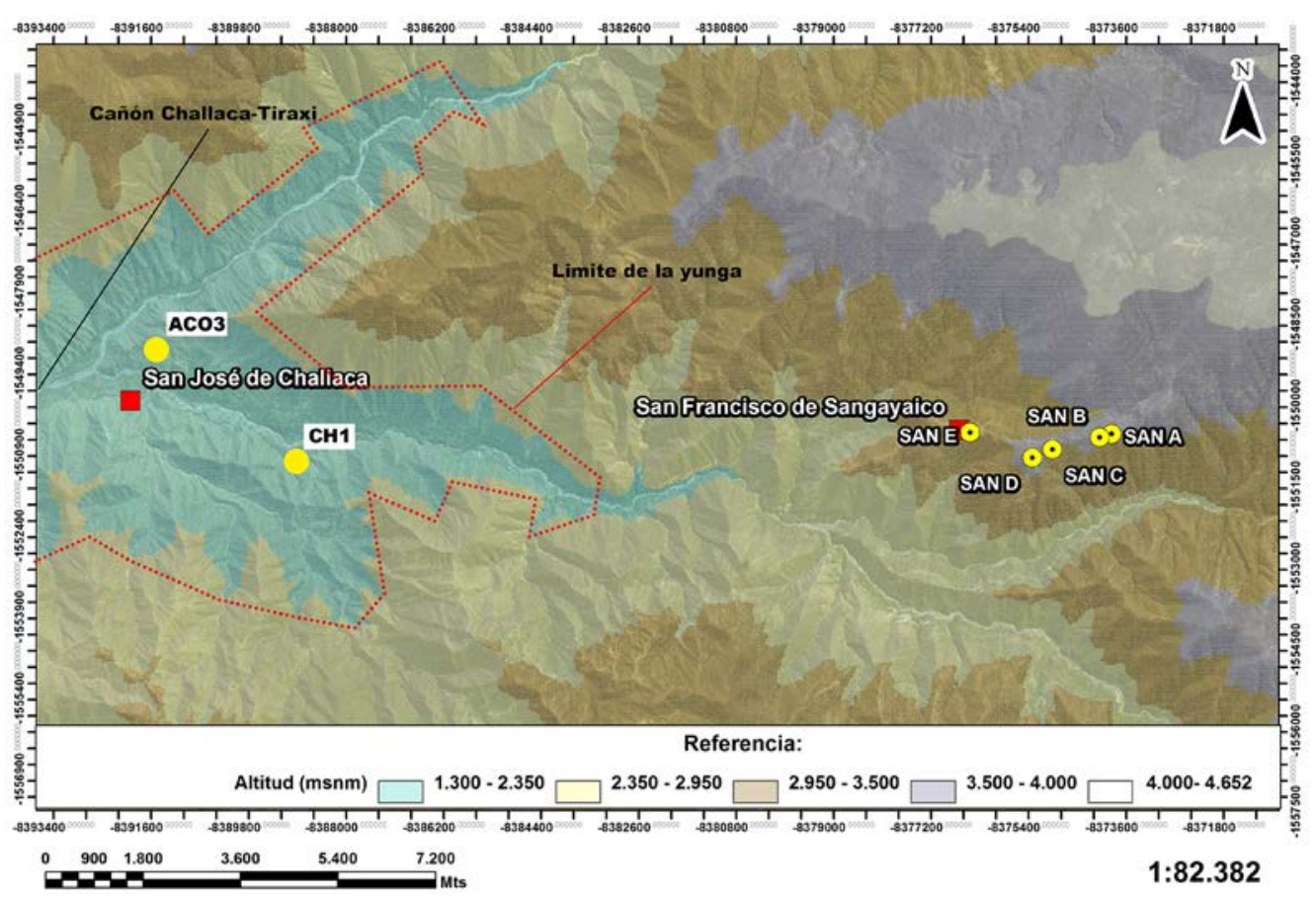

Figura 2. Mapa de ubicación de los sitios $\mathrm{CH} 1$ y ACO3, mostrando limite de la yunga y el cañón Challaca-Tiraxi (ORP-PIACI 2014).

terrazas se encuentran principalmente localizadas hacia el norte; y, en ACO3, hacia el este. Sobre la base de su tamaño relativo, la falta de registro que indique la existencia de redes de canales de riego y la presencia de posibles pisos de ocupación enterrados (descubiertos durante las excavaciones en $\mathrm{CH} 1$, ver más abajo), interpretamos que estos tuvieron una función habitacional en lugar de terrazas productivas. Sin embargo, se necesitan más estudios para corroborar por completo esta funcionalidad. Por otro lado, aparte de la ubicación elevada de $\mathrm{CH} 1$ y de ACO3, no hay características defensivas fácilmente identificables. Hasta el momento, de los dos sitios, solo Challaca $(\mathrm{CH} 1)$ fue excavado y se registró una ocupación sólida nasca, que está estratigráficamente ubicada por arriba de una ocupación más efímera del Horizonte Temprano.

\section{Excavaciones en Challaca (CH1)}

En el sitio Challaca (CH1, Fig. 3, aproximadamente 2015 metros sobre el nivel del mar), no hay ninguna evidencia arqueológica en superficie sobre las faldas este y oeste del cerro, posiblemente, por el hecho de la inclinación de las laderas. La ladera norte - hacia el fondo del valle- está parcialmente resguardada por un espolón de roca que se extiende desde el oeste. Este espolón comprende el límite oeste del sitio, mientras que otro espolón, más chico, delimita el este. La superficie del sitio está cubierta por una escasa vegetación, que se compone mayormente de arbustos y cactus. En el sitio, domina visualmente un área de cultivo que se extiende desde este sector hacia el oeste hasta el límite con el cañón de Challaca-Tiraxi.

El sitio presenta una abundante evidencia sobre posibles estructuras domésticas con material arqueológico diagnóstico y no diagnóstico. La cerámica de superficie sugiere una ocupación nasca con algún material paracas (estilo incisivo del Horizonte Temprano/Formativo). La plataforma superior está caracterizada por una visible estructura grande circular (aproximadamente ocho 


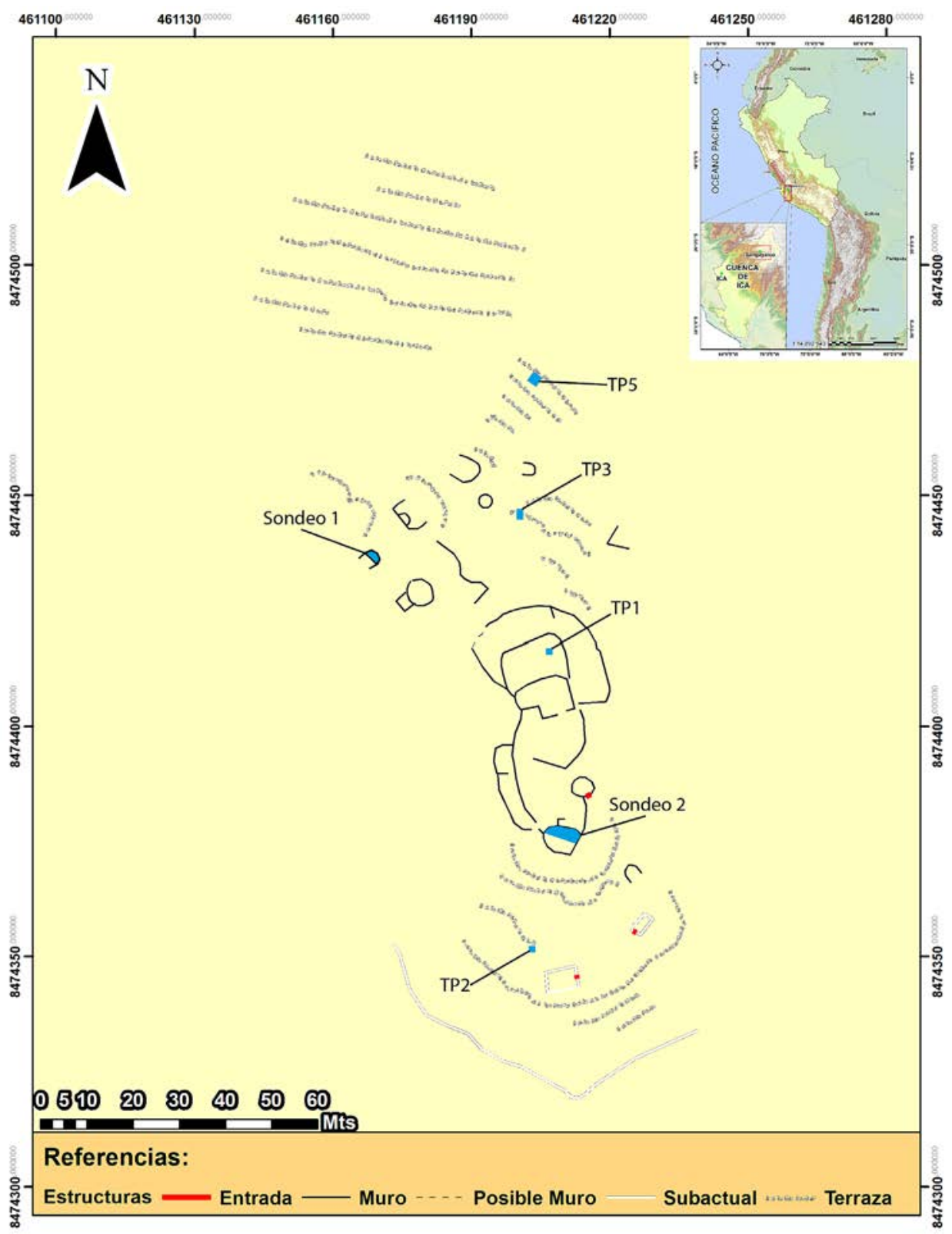

Figura 3. Plano de CH1, mostrando unidades de excavación (ORP-PIACI 2014).

metros de diámetro), en la que se puede observar solamente una hilera. Hacia el este y oeste de esta cima, y de la estructura circular, se identificaron otras estructuras más chicas en forma de «D». Posiblemente, la construcción de estas estructuras significó la nivelación artificial de los sectores del sitio, al igual que la construcción de las terrazas.

La plataforma superior presentó muy poca estratigrafía arqueológica, dado que principalmente estaba compuesta por un depósito limoso de poca profundidad sobre la roca madre. Además, no se halló ningún horizonte de ocupación evidente, puesto que la superficie estaba muy bioturbada al igual que disturbada por procesos posdepositacionales de ente humana. Por otro lado, hubo una mayor acumulación de material sobre la ladera norte del sitio (v.g. TP 3 y 5 - ver abajo), debido a 
la existencia de terrazas en esta área y su subsecuente colapso. En total, se excavaron seis sondeos de prueba de diferentes tamaños a lo largo del sitio. A continuación, se detalla brevemente las diferentes unidades de excavación.

\subsection{Challaca (CH1): Sondeo (Pit) 1}

El objetivo de este sondeo fue registrar los elementos asociados a una estructura (muro de piedra) de forma semicircular. El Sondeo 1 está ubicado en el extremo noroeste del sitio sobre un espolón, desde donde se vislumbra el río Santiago hacia el norte. En sí, el sondeo se encuentra dentro de una posible estructura subcircular de cuatro metros de norte a sur por 3, 8 metros de este a oeste. El muro de la estructura tiene doble cara (con 0,45 a 0,55 metros de ancho) y está muy deteriorado, especialmente, en el lado oeste. Durante las excavaciones, se retiraron dos capas. La primera corresponde al desmonte que se acumuló luego del abandono. La segunda capa removida fue la tierra que se acumuló con la finalidad de asentar el muro de piedra que define la estructura.

El sondeo cubrió cerca del 50 por ciento del área interior de la edificación. Durante la excavación, se recolectaron fragmentos de cerámica cuya decoración se asocia a las figuras y el colorido del estilo Nasca 3-4 y 7 propio de la costa. Esto nos sugiere que la edificación y el uso de la estructura se habrían realizado al menos entre el 440 y el 640 d.C.

Si consideramos lo antes dicho, en relación con el hecho de que el área intervenida se emplaza sobre un espolón que permite tener un dominio visual sobre el lado norte del río Santiago, podríamos proponer que la estructura tuvo como propósito el rol de punto de «control» visual del área adyacente. Por otro lado, esta inferencia sobre la funcionalidad de un lugar de vigilancia u observación es coherente con las características de la arquitectura presente y los contextos excavados.

\subsection{Challaca (CH1): Sondeo (Pit) 2}

En el sitio Challaca, el área que cuenta con mayor espacio para ser usado es la cima. La parte superior del cerro fue modificada y acondicionada con muros para generar espacios de mayores dimensiones a las terrazas presentes en la ladera norte. También, la cima es la zona más afectadas por los procesos posdeposicionales. Los muros de los recintos se encuentran muy disturbados y cubiertos por sus mismos componentes (bloques de piedra). A pesar de ello, es posible advertir que, en la parte alta, se ubicó una edificación de planta irregular. Esta estructura contó con varios «recintos» comunicados, algunos de ellos a desnivel. Estos últimos estuvieron emplazados en el lado norte de la cima. Para esclarecer la funcionalidad de los recintos, se decidió ubicar el Sondeo 2 en el extremo sur de la estructura. Este sondeo se ubica en la parte más alta del sitio y sus dimensiones (cuatro metros por seis metros) cubren la mitad norte de una posible unidad habitacional semicircular o en forma de «D». Esta estructura está directamente asociada a otra estructura circular hallada en la cima del cerro Challaca. Luego de retirar la capa de escombros, al norte, se identificó una especie de banqueta. En la esquina noroeste y noreste del sondeo, se ubican dos cortes de forma «circular» de 80 centímetros de diámetro; estos cortes se hicieron sobre la roca madre. Ambos «hoyos» estuvieron rellenados con ceniza, restos de moluscos y huesos. También, se registró una vasija completa en la base del muro noroeste.

En la excavación, se recolectó una gran cantidad de cerámica diagnóstica, tanto en el interior de los escombros como en el interior de las capas acumuladas durante la construcción del edificio. Precisamente, en estos últimos contextos, se recuperaron tiestos de evidente filiación al estilo Nasca 7, lo que sugeriría una probable contemporaneidad con la estructura subcircular hallada en el Sondeo 1. Además, la presencia de tiestos de estilo Nasca 7 fecha al recinto con ocupaciones asociadas al Período Intermedio Temprano tardío, mientras que otras piezas del Nasca 3 y 4 nos aportan datos sobre una continuidad de ocupación desde el comienzo de Nasca (c. 80 a.C.).

Por otro lado, el recinto cuenta con un diseńo poco usual para un área doméstica. Por ejemplo, hacia los extremos del muro curvo, se cuenta con una depresión de planta cuadrangular a desnivel. 


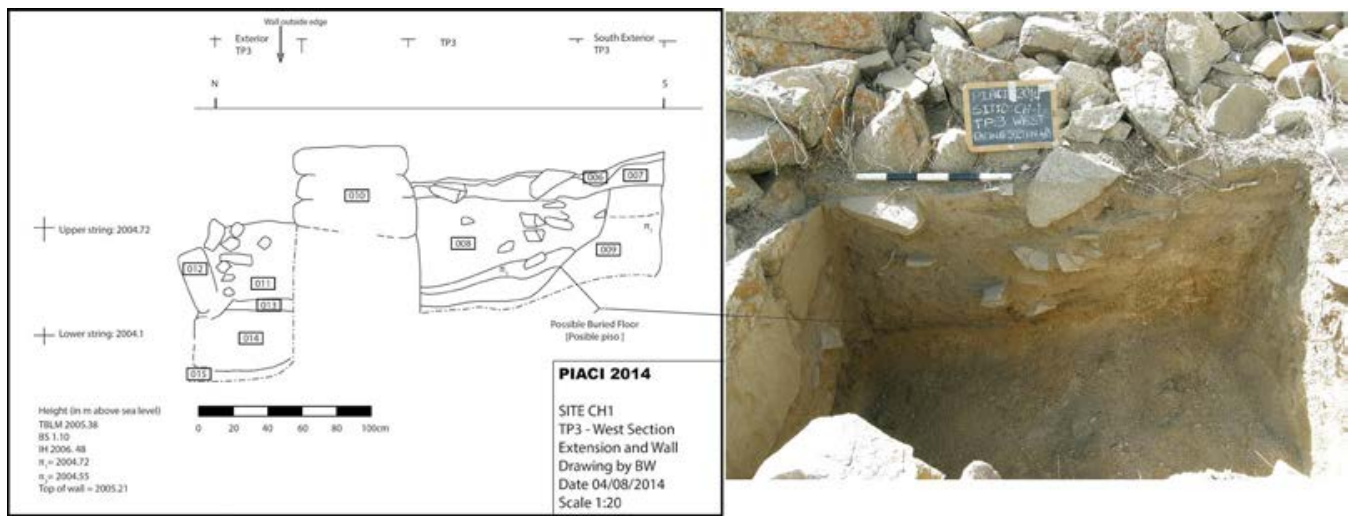

Figura 4. Perfil de TP3, mostrando posible piso (ORP-PIACI 2014).

Esto indica que la zona fue edificada como un lugar de carácter «especial». Además, esta inferencia es congruente con el registro de concentraciones de cenizas - posibles restos de quemas asociadas con la consagración del lugar- y la colocación de una vasija completa debajo del muro curvo.

\subsection{Challaca (CH1): Test-Pit 1 (TP1)}

El sondeo de prueba (Test-Pit) ubicado en el flanco norte de la cima del sitio dentro de una posible estructura subcircular no brindó material diagnóstico.

\subsection{Challaca (CH1): Test-Pit 2 (TP2)}

Este sondeo de prueba (Test-Pit) se ubicó en el flanco sur sobre una terraza moderna que se encuentra hacia el norte de una estructura moderna junto a la actual carretera. No se encontró registro arqueológico de entes diagnósticos, dado que el material era totalmente reciente.

\subsection{Challaca (CH1): Test-Pit 3 (TP3)}

Además de excavar en el sitio de Challaca $(\mathrm{CH} 1)$, se realizó la prospección sistemática de superficie. Sobre la base del registro de los vestigios arqueológicos, se pueden distinguir dos áreas: la cima, que fue descrita anteriormente, y la ladera norte. El flanco norte del sitio de CH1 está compuesto por una serie de aterrazamientos que van desde la cima del sitio hasta el río. Estas terrazas son irregulares, por lo que varían entre dos y tres metros de ancho, y de cuatro a cinco metros de extensión, con una forma ligeramente curva que sigue los contornos de la colina. Es pertinente mencionar que la ladera este tiene un perfil empinado, por lo que es de difícil acceso. En aquella área de perfil empinado, solo se apreciaron restos arquitectónicos cerca de la cima y las proyecciones de las terrazas que vienen desde la ladera norte. Precisamente, para establecer la temporalidad y el carácter de la arquitectura, se ubicaron dos sondeos de excavación: Test-Pit 3 y Test-Pit 5.

El Sondeo 3 está sobre el extremo este de la tercera línea desde la cima de las terrazas existentes sobre la ladera norte. El sondeo se encuentra en una terraza que se emplaza cerca a la parte superior de la ladera norte del sitio. El sondeo se extendió por delante y detrás de la terraza. Hacia el sur de la terraza, TP3 mide 160 centímetros por 100 centímetros, mientras que delante de la terraza, hacia el norte de la estructura, el sondeo mide 50 centímetros por 50 centímetros.

En principio, se interpretó que las terrazas fueron edificadas y utilizadas como unidades domésticas. Esta inferencia fue establecida sobre la base del registro de una mesa de moler in situ y algunas manos de moler registradas entre la arquitectura conservada. Además, se asocian abundantes 
fragmentos de tiestos de cuencos y vasijas para la elaboración, preparación y consumo de alimentos. Por otro lado, en el material de superficie, se registraron tiestos decorados que se asocian a los estilos cerámicos culturales, Nasca 3, 4 y 7, y algunos fragmentos podrían estar asociados al Horizonte Temprano, debido a la presencia de cerámica de estilo Ocuaje 8 (380 a 260 d.C.) Por consiguiente, las terrazas probablemente corresponderían a una ocupación inicial del Horizonte Temprano (Paracas/Ocucaje) antes de pasar al Intermedio Temprano (Nasca).

La estratigrafía registrada en Sondeo 3 permite afirmar que por debajo de las terrazas no se ubican otras estructuras. Por otro lado, detrás de los muros de contención se acumuló varios tipos de rellenos postabandono. Luego de excavar los escombros (una capa de 10 centímetros), se retiró cuatro capas de relleno de diferente composición. Dentro del relleno acumulado sobre el nivel estéril, se registraron concentraciones de carbón y ceniza. La baja densidad de las concentraciones y la ausencia de manchas de quema nos indican que los restos encontrados fueron trasladados, por lo que no serían adecuados para datar el momento de la construcción de la terraza.

Aproximadamente, a los 60 centímetros por debajo de la actual superficie se hallaron restos de un posible piso de limo arenoso con concentraciones de cenizas con carbón (Fig. 4). En el relleno, se encontraron unos pocos fragmentos de cerámica de estilo Nasca 7, lo cual nos da una posible estimación del momento de abandono de las edificaciones. Podemos afirmar que la terraza donde se ubica Sondeo 3 junto con las otras terrazas de la ladera son las únicas arquitecturas edificadas para acondicionar un área habitacional; es decir, solo hay una fase de construcción que fue subsecuentemente usada durante el Horizonte Temprano e Intermedio Temprano.

\subsection{Challaca (CH1): Test-Pit 5 (TP5)}

El sondeo estuvo ubicado en el extremo este de una de las terrazas que se localizan en la parte media de la ladera norte del sitio arqueológico (similar a TP3). La excavación del TP5 nos permitió identificar que, dentro de los rellenos constructivos acumulados para la edificación de las terrazas, se colocaron huesos de animales y depósitos secundarios de algunos restos óseos humanos que podrían haber sido posibles ofrendas.

El TP5 fue el segundo intento que se realizó con la finalidad de corroborar o refutar la hipótesis de trabajo establecida con la prospección de la ladera norte. También, se orientó a corroborar si las terrazas eran las primeras y únicas edificaciones construidas para acondicionamiento habitacional. La terraza seleccionada se encuentra en la parte media de la ladera, y, debajo de ella, los muros han colapsado cuesta abajo o solo conservan algunos bloques de piedra de la base. La terraza mide entre tres y cuatro metros de ancho, y más de 10 metros de largo. El muro de gravedad que define la superficie horizontal se proyecta hacia arriba de forma de muro medianero.

La excavación del piso de ocupación proporcionó los mismos resultados que los registrados en el TP3, es decir, un área muy disturbada por el crecimiento de raíces de vegetación moderna, y la ausencia de conjunto de artefactos o restos de otros materiales asociados. Contrario a lo sucedido en el TP3, los rellenos constructivos de la terraza proporcionaron evidencia muy interesante. A poco menos de 20 centímetros de la superficie del relleno constructivo, se identificó un primer pequeño «anillo» circular de piedra (Fig. 5). El anillo está compuesto de piedras superpuestas que forman un tipo de "caja»; visto de planta tiene forma ovalada o de anillo. Para construir este anillo de piedras, fue necesario cortar parte de la capa estéril que se ubica por debajo de la unidad estratigráfica. El anillo tiene en realidad forma de apacheta chata y se edificó superponiendo dos niveles de piedras. Las piedras se han adosado usando arcilla arenosa como mortero. Este anillo mide 55 centímetros de este a oeste por 65 centímetros de norte a sur y alcanza 48 centímetros de altura. Las lajas utilizadas son de tamaño mediano y sus dimensiones varían entre 15 y 20 centímetros de largo por 12 a 15 centímetros de ancho, y 6 centímetros de alto.

En el interior de este anillo, se detectó un relleno de tierra arcillosa junto a óseos de animales (camélidos) y óseos humanos (fémur, costillas, vértebras). En la base de este pequeño anillo circular, a unos 20 centímetros hacia el norte, se reconoció la presencia de un cráneo humano, el cual estaba 


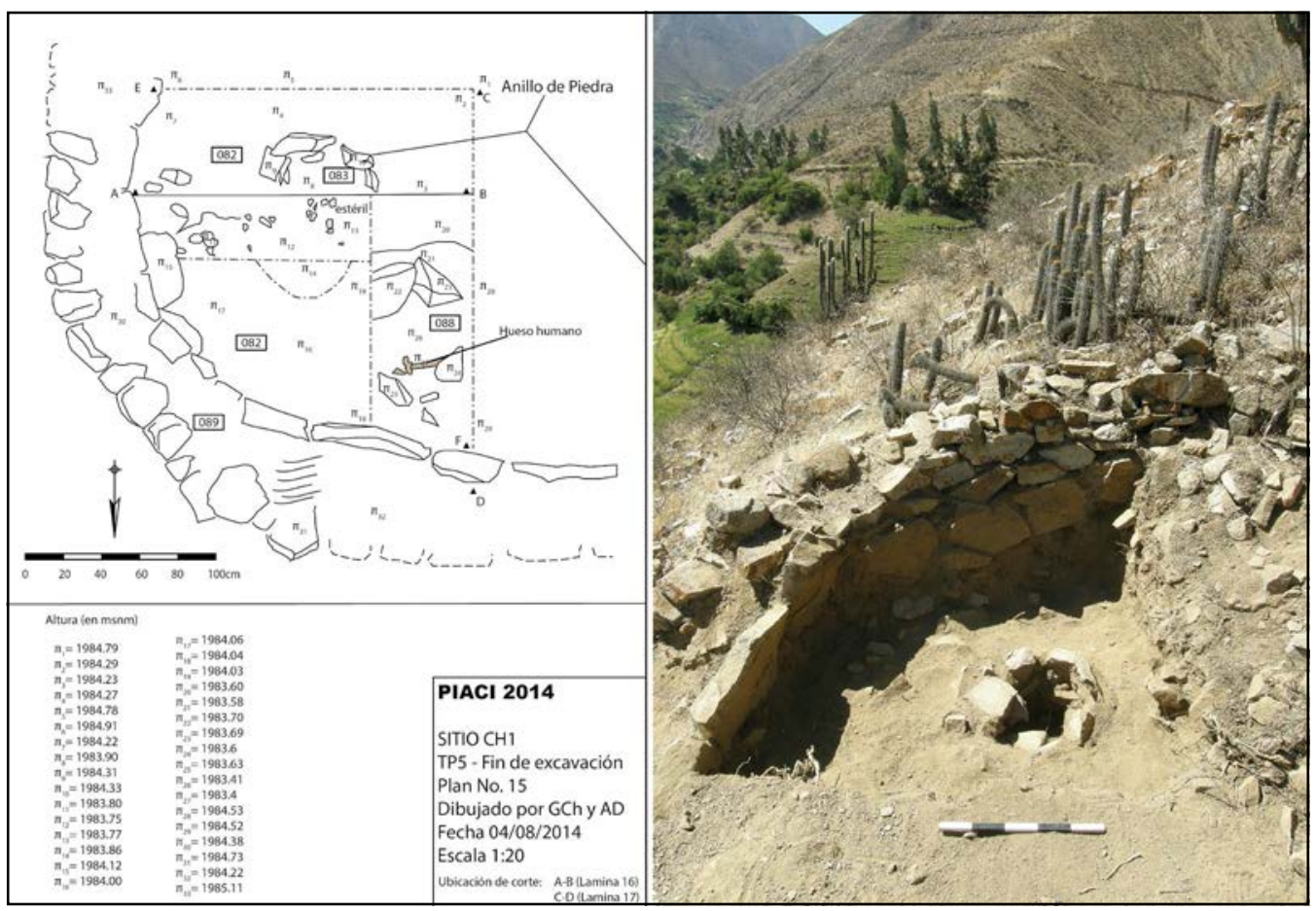

Figura 5. Plano de TP5, mostrando «anillo» de piedras (ORP-PIACI 2014).

asociado a dos primeras vértebras que se encontraban cerca del orificio occipital. Rodeando al cráneo y en la cúpula se registró ceniza, la cual podría ser resultado de alguna quema efectuada antes de depositar el cráneo y rellenar el anillo circular.

Debajo de la base interior del muro de contención, y cubierto por relleno constructivo, se halló un segundo anillo circular también relleno con tierra y huesos humanos. Este anillo no fue excavado en su totalidad y se proyecta al interior del perfil oeste del TP 5, por lo que solo cuatro bloques que miden 20 centímetros por 20 centímetros fueron expuestos. Al igual que el primer anillo, las piedras fueron asentadas directamente sobre el estéril. Durante la excavación, se observó que la tierra que rellenaba este segundo anillo era de color marrón con una tonalidad media a oscura. También, se observó grava pequeña y angulosa abundante en la parte superior del anillo. La cerámica asociada a los dos anillos circulares corresponde a tiestos de ollas de color gris, sin cuello y decorado con líneas incisas en forma de triángulos o círculos con puntos al centro - cerámica indudablemente del Horizonte Temprano (Ocucaje 8, Fig. 6)—.

A partir de lo descrito anteriormente, se puede confirmar que en la ladera norte solo tuvo un momento de construcción. Por otro lado, la presencia de cerámica monocroma, propia del Horizonte Temprano, permite estimar que la construcción de las terrazas sucedió durante ese período o poco tiempo después. Además, los depósitos encontrados dentro de las estructuras circulares podrían ser interpretados como ofrendas dedicadas a la construcción de estas estructuras habitaciones sobre terrazas.

En TP5, no se pudo ubicar un piso de ocupación arqueológico dado el grado de bioturbación de los rellenos y depósitos en ella. De todas formas, el material mixto hallado en los rellenos del Horizonte Temprano e Intermedio Temprano nos sugiere que, al igual que TP3, estas terrazas fueron ocupadas por lo menos en estos dos períodos. 


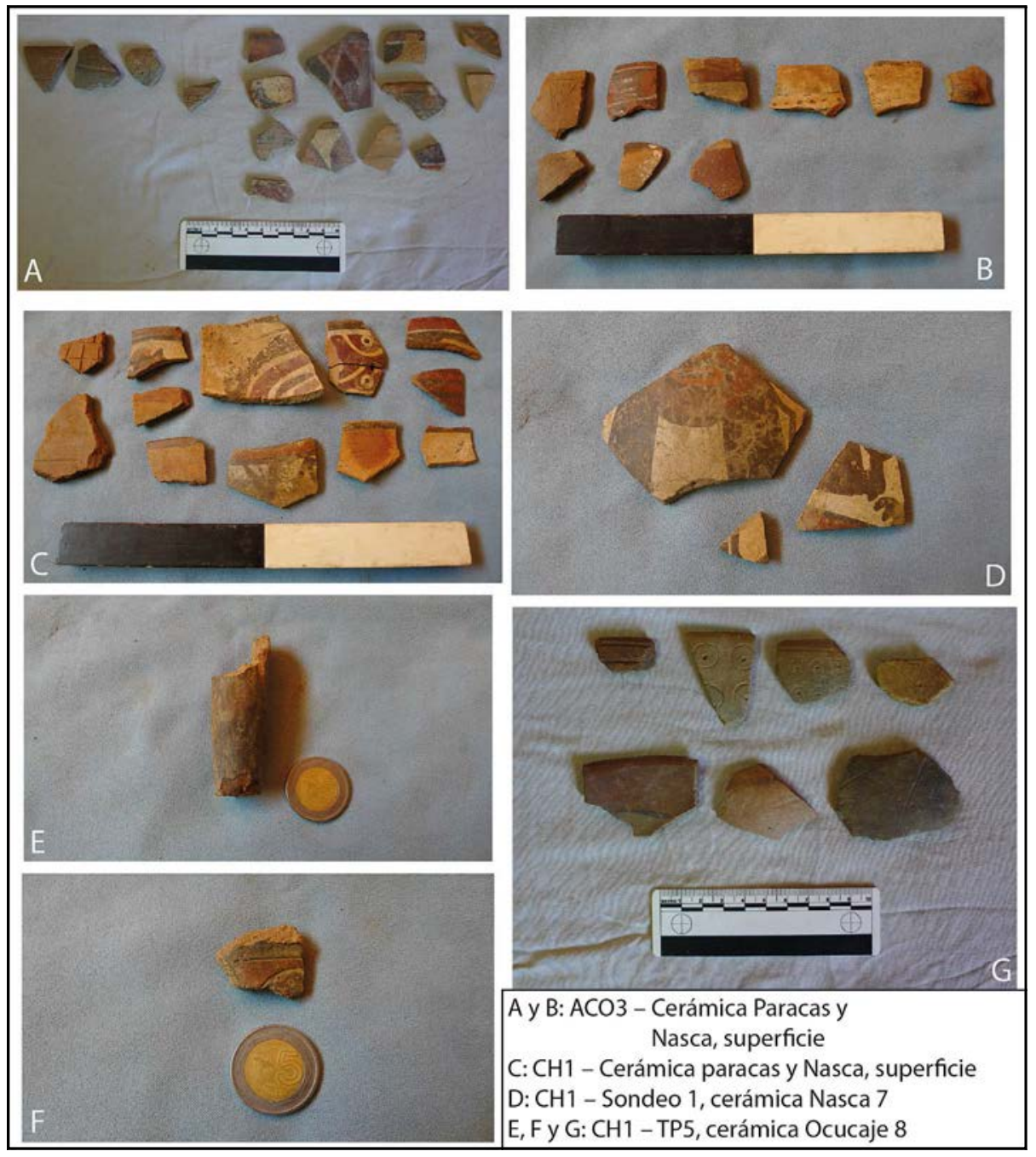

Figura 6. Cerámica Paracas y Nasca de ACO3 y CH1 (ORP-PIACI 2014).

\section{Discusión}

Nuestras prospecciones iniciales han confirmado que Challaca $(\mathrm{CH} 1)$ y Cerro San Bernardo (ACO3) tienen evidencias de ocupaciones con cronologías ubicadas durante el Horizonte Temprano y del Período Intermedio Temprano. Sobre todo, las excavaciones en CH1 nos permitieron evaluar la intensidad de estas ocupaciones. Por otro lado, el hecho de que el asentamiento Nasca había subsumido al Paracas/Ocucaje 8 sugiere que podría haber existido una continuidad en la ocupación del sitio que conecta estos dos períodos. Las posibles ofrendas halladas en CH1/TP3 hacen hincapié en una fase inicial Paracas/Ocucaje 8 en el sitio. Sin embargo, todavía no es posible delimitar claramente las características de esta ocupación del Horizonte Temprano en el sitio, dada la escasez de los restos materiales de este período. Por lo tanto, nuevas excavaciones serán necesarias para desentrañar el entramado de esta ocupación temprana, y sus vínculos — si es existieran — con la ocupación del período Nasca Tardío. 
El asentamiento nasca en $\mathrm{CH} 1$, a pesar de los altos impactos generados por los procesos posdepositacionales, brinda una información suficientemente clara para formular importantes interpretaciones preliminares sobre el sitio y su contexto, especialmente, cuando se combina con los datos provenientes del Cerro San Bernardo (ACO3). Más allá de la localización de estos sitios, en la parte superior de los montículos, estos dos asentamientos nasca no presentan ninguna estructura discernible de protección o semejantes. No obstante, esto no excluye la naturaleza defensiva de estos asentamientos, especialmente, teniendo en cuenta que el final del Horizonte Temprano y del Período Intermedio Temprano ha sido considerado como un momento con abundante conflicto, especialmente, entre las culturas del Intermedio Temprano (Arkush y Tung 2013).

Por otro lado, la importancia defensiva de estos sitios está más enfatizada por el aislamiento relativo de estos con respecto a la costa central del valle de Ica, por lo que para la región esta área habría tenido un valor atípico o de alguna funcionalidad de colonia. Si bien se acepta que durante Nasca 7 fue el momento en el cual se produjo la mayor interacción nasca en las tierras altas (Silverman 2002: 178; Whalen 2014), especialmente con Huarpa del área circum-Ayacucho (Knobloch 2000; Leoni 2006), la naturaleza de estos contactos todavía no ha sido definida. Aparte de eso, la presencia de cerámica Nasca 3 y 4 (Figs. 6A y C; c. 80 a.C. a 440 d.C.) nos sugiere que estos contactos con la sierra se remontan a períodos aún más tempranos también. En los sectores bajos, yunga, de las tierras altas del valle de Ica, estas relaciones parecen haber estado circunscritas con un propósito por parte de los Nasca para mantener su presencia en la región.

De esta forma, los sitios de Challaca (CH1) y Cerro San Bernardo (ACO3) dominan el este del área que se trata de un rico bolsón productivo ubicado en el cañón de Challaca-Tiraxi (Fig. 7). Probablemente, esta "colonia» Nasca fue un intento por parte de esta cultura de extender su control hacia la sierra y así tener acceso directo a los productos del área — maíz, coca, frutales—al igual que servir como punto de partida para intercambiar productos con las poblaciones del kichwa y puna, tal como fibra de camélidos y la papa.

También, es posible que esta zona haya funcionado como nexo para el desarrollo de un pastoreo incipiente de camélidos para la sociedad Nasca. El proyecto alemán Palpa-Nasca, liderado por Marcus Reindel, ha demostrado cómo los sitios en estas altitudes podrían haber funcionado dentro de una red de interacción entre las tierras bajas costeras con el pastoralismo de llama de las tierras altas (Renneberg et al. 2009). Este pastoreo de llamas bien podría haberse beneficiado con la explotación de las cuatro zonas ecológicas disponibles para los momentos finales de Nasca - las riberas de los fondos del valle, la zona litoral costera, las lomas y yungas de las zonas más bajas de las tierras altas-; de este modo, se habría cubierto un área que abarcaría desde las zonas con altitud a nivel del mar hasta zonas con mayores altitudes ejemplo, 2300 metros. De hecho, habríamos tenido un tipo de archipiélago vertical compacto de acopio de diferentes productos a través de estas cuatro zonas ecológicas (Murra 1975 [1972]; sensu Oberem 1976).

En este sistema, los camélidos podrían haber sido alimentados con maíz o tallos de maíz después de su pastoreo, con las pasturas existentes entre los meses de abril y agosto (sensu Miller y Burger 1995); esta dieta puede haber sido complementada con vainas de huarango (Prosopis limensis) y «uñas de gato» (Parkinsonia aculeate), además de otras plantas. Al mismo tiempo, el guano de los camélidos habría sido un fertilizante natural para los campos de cultivos que estaban en período de barbecho. A su vez, estos oasis de ribera habrían conformado corredores de tránsito hacia zonas del litoral para el consumo por parte del rebaño del seagrass propio de la zona costera. Entre los meses de agosto y diciembre, los rebaños se habrían movido a través de estos corredores conectando las yungas de la tierra alta, antes de bajar a las lomas para el período comprendido entre diciembre y abril. Este patrón de trashumancia yunga-lomas-fondo de valle-litoral costero es una reminiscencia de la sugerida para los Moche (Dufour et al. 2014). Hasta hace poco, dicha trashumancia fue realizada por los pastores de cabras y ovejas en el valle de Ica (Whaley et al. 2010: 619-620), y sectores más al norte, como Piura (Perevolotsky 1985). 


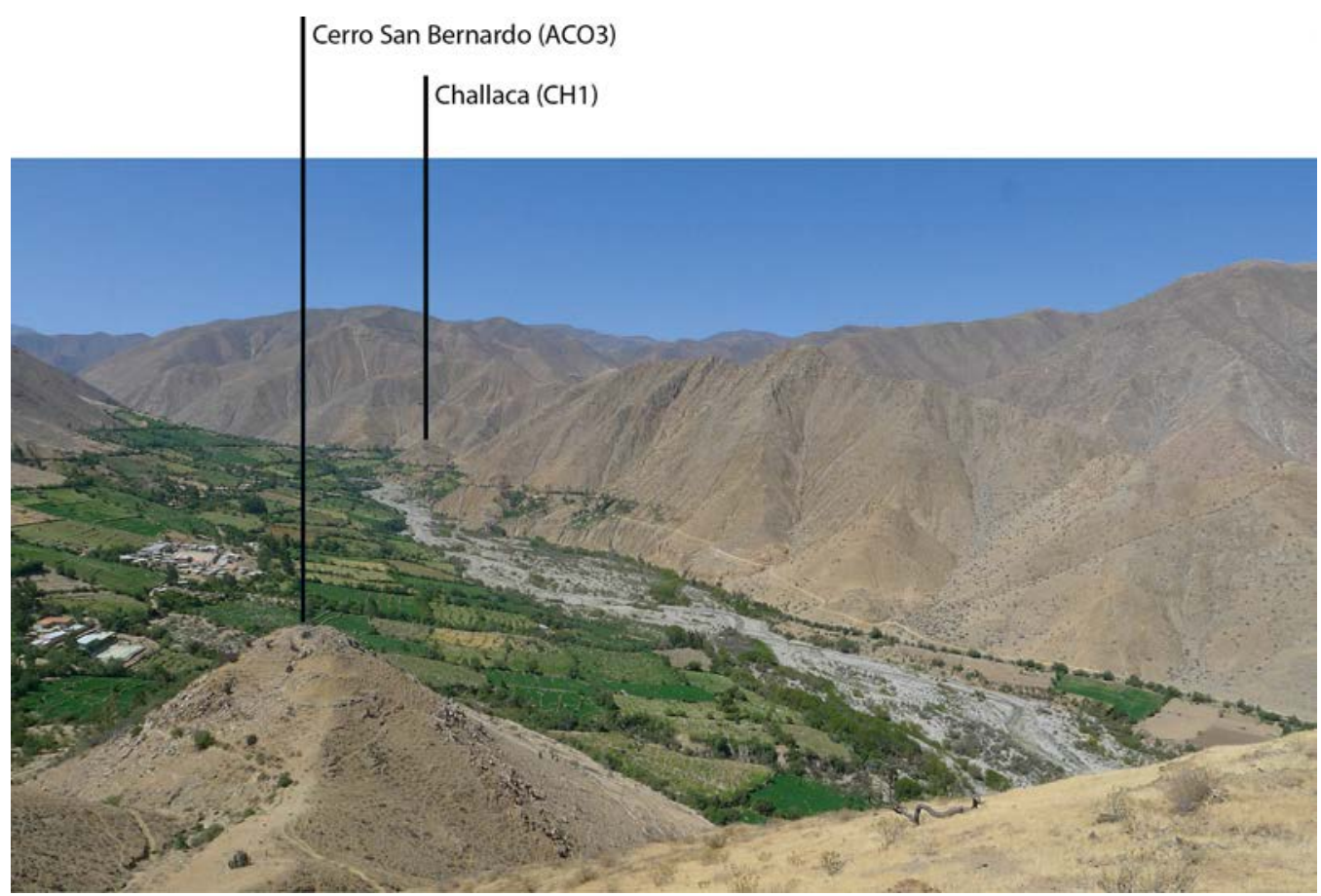

Figura 7. Panorámica moderno del enclave Nasca, mostrando los sitios de ACO3 y CH1 (ORP-PIACI 2014).

De este modo, estas «colonias» Nasca de Challaca (CH1) y Cerro San Bernardo (ACO3), localizadas a aproximadamente 2000 metros sobre el nivel del mar, en la cuenca alta de Ica, podrían bien haber provisto una frontera logística para el control Nasca en la región yunga costera y en las tierras altas medias, sin dejar de proveer el medio necesario para el intercambio y el contacto con los sectores superiores de las tierras altas. De hecho, la ubicación de estos sitios en los límites de las ecozonas yunga y kichwa nos sugiere la posibilidad de que $\mathrm{CH} 1$ y ACO3 hayan sido sitios de avance con control de acceso directo a la zona central de los Nasca en la Ica central. La ubicación sobre espolones rocosos en ambos lados del mismo río posiblemente conformaba parte de una frontera geopolítica fluida, pero al mismo tiempo estable de la cultura Nasca vis-à-vis los pueblos de la sierra. La ausencia de material del Horizonte Medio nos podría indicar el abandono de estos sitios antes de la aparición de los Wari, posiblemente asociado a cambios sociopolíticos y económicos en la zona costera (Beresford-Jones 2011).

$\mathrm{Al}$ mismo tiempo, el material recuperado de las excavaciones en Challaca $(\mathrm{CH} 1)$ nos muestra que esta penetración costeña podría tener una antigüedad aún mayor, lo cual indica una posible ocupación del área desde el Horizonte Temprano (840 a 260 a.C) en adelante. El hecho de la existencia de estructuras similares, aunque de mayor envergadura, descubiertas en el Alto Palpa nos muestra que esta posible expansión costeña hacia la sierra no fue necesariamente la excepción, sino parte de un patrón más amplio macrorregional (Reindel et al. 2015; Sossna 2016).

El posterior establecimiento de sitios del Intermedio Tardío (ACO1, 2, 4, 5; CH2, CH3, CH5, CH10-13; RUR1) en las laderas ubicadas encima de los sitios de Challaca (CH1) y Cerro San Bernardo (ACO3) nos proporciona evidencia sobre la recurrente importancia de esta zona fértil para las poblaciones del área. No obstante, a diferencia de Challaca $(\mathrm{CH} 1)$ y Cerro San Bernardo (ACO3), la cultura material de estos otros sitios nos indica que, para estos momentos, no era la costa la que ejercía hegemonía, sino más bien los pobladores de la sierra, en este caso, los Chocorvos (Rowe 1946: 188). 


\section{Conclusiones}

En esencia, la presencia de material del Horizonte Temprano costero - Paracas - y la del Período Intermedio Temprano - Nasca - en estos dos sitios da cuenta de la posibilidad de algún tipo de límite geopolítico entre los Nasca y las entidades políticas contemporáneas de las tierras altas, como los Huarpa (Schreiber 2000). Mientras que Paracas costera encuentra una contraparte no muy diferente en la sierra (Ochatoma Paravicino 1998), el Intermedio Temprano está marcado por diferentes estilos de cerámica que denotan entidades políticas separadas. Posiblemente, en esta etapa, la naturaleza de estos sitios entra al frente, dentro de un área que sirve como lugar de una "geografía difusa» (Haughton y Allmendinger 2013: 218) entre la costa y los sectores superiores de las tierras altas, donde el intercambio y las interacciones ocurren tanto de forma violenta como pacíficas.

Teniendo en cuenta, como se ha mencionado anteriormente, que la distribución interna de la arquitectura de los sitios comparte similitudes con los sitios identificados contemporáneos en el valle superior de Palpa (Reindel 2012; Reindel et al. 2013; Sossna 2016), se plantea la posibilidad de que este tipo de interacción costera basada en la ocupación real de los sectores de las tierras altas fuera replicada a lo largo de varios valles costeros. De hecho, estas dinámicas de asentamiento reflejan los diversos factores políticos, sociales, económicos y ecológicos que subyacen el florecimiento, el declive (o colapso) y la regeneración de las sociedades (Schwartz y Nichols 2006). Por esta razón, el presente estudio ha puesto de manifiesto la profundidad de la ocupación y la historicidad de uso humano de esta zona.

\section{Agradecimientos}

Extendemos nuestros agradecimientos a las comunidades de Santiago de Chocorvos, San José de Challaca y Santa Rosa de Acora. También, agradecemos el trabajo de traducción y edición de Lorena Grana; el trabajo final hubiera sido mucho más pobre sin su ayuda. Agradecemos profundamente a todos los participantes del proyecto. Finalmente, queremos agradecer también a Jalh Dulanto y Aïcha Bachir Bacha por su paciencia. Todos los errores permanecen de los autores.

El financiamiento para este proyecto fue proporcionado por la Leverhulme Foundation y Don Alberto Benavides. El proyecto se llevó a cabo bajo el auspicio del Ministerio de Cultura del Perú y las resoluciones 061-2013-DGPA-VMPCIC/M y 428-2014-DGPA-VMPCIC/MC.

\section{REFERENCIAS}

\section{Arkush, E. y T. A. Tung}

2013 Patterns of war in the Andes from the Archaic to the Late Horizon: insights from settlement patterns and cranial trauma, Journal of Archaeological Research 21 (4), 307-369. https://doi.org/10.1007/s10814-0139065-1

Bachir Bacha, A. y O. D. Llanos

2013 ¿Hacia un urbanismo Paracas en Ánimas Altas/Ánimas Bajas (valle de Ica)? A. Bachir Bacha y J. Dulanto Brescia (eds.), Paracas: nuevas evidencias, nuevas perspectivas, Boletín de Arqueología PUCP 17, 169-204.

Beresford-Jones, D. G.

2011 The lost woodlands of Ancient Nasca: a case-study in ecological and cultural collapse, The British Academy/ Oxford University Press, Oxford. https://doi.org/10.5871/bacad/9780197264768.001.0001

Beresford-Jones, D. G., A. G. Pullen, O. Q. Whaley, J. F. Moat, G. Chauca, L. Cadwallader, S. Arce, A. Orellana, C. Alarcón, M. Gorriti, P. Maita, F. Sturt, A. Dupeyron, O. Huaman, K. J. Lane y C. A. French

2016 Re-evaluating the resource potential of lomas fog oasis environments for preceramic Hunter-Gatherers under Past ENSO modes on the South Coast of Peru, Quaternary Science Reviews 129, 196-215. https:// doi.org/10.1016/j.quascirev.2015.10.025 


\section{Chauca Iparraguirre, G. y K. Lane}

2015 Informe final: proyecto de investigación arqueológica de la cuenca de Ica [PIACI] - Temporada 2014, Dirección General de Patrimonio Arqueológico Inmueble, Ministerio de Cultura, Lima.

Cook, A. G.

1991 Informe final de las invesitigaciones de reconocimiento en la parte baja del valle de Ica, 1988-1990, informe presentado al Instituto Nacional de Cultura, Lima.

Dufour, E., N. Goepfert, B. Gutiérrez Léon, C. Chauchat, R. Franco Jordán y S. Vásquez Sánchez

2014 Pastoralism in northern Peru during pre-Hispanic times: insights from the Mochica Period (100-800 AD) based on stable isotopic analysis of domestic camelids, PLoS One 9 (1). https://doi.org/10.1371/ journal.pone.0087559

Engel, F.

1957 Early sites on the Peruvian coast, Southwest Journal of Anthropology 13 (1), 54-68. https://doi.org/10.1086/ soutjanth.13.1.3629157

French, C., D. Beresford-Jones y K. Lane

2012 Changes in ancient land and water-use along the Río Ica, South-Central Andes, Leverhulme Large Research Project, University of Cambridge, Cambridge.

\section{Haughton, G. y P. Allmendinger}

2013 Spaces of neoliberal experimentation: soft spaces, postpolitics, and neoliberal governmentality, Environment and Planning A 45, 217-234. https://doi.org/10.1068/a45121

Huamán Oros, O. y K. Lane

2014 Informe Final: Proyecto de investigación arqueológica de la Cuenca de Ica [PIACI] - Temporada 2013, Dirección General de Patrimonio Arqueológico Inmueble, Ministerio de Cultura, Lima.

Knobloch, P. J.

2000 La chronología del contacto y encuentros cercanos de Wari, en: P. Kaulicke y W. H. Isbell (eds.), Huari y Tiwanaku: Modelos versus evidencias. Primera parte, Boletin de Arqueología PUCP 4, 69-88.

Leoni, J. B.

2006 Ritual and society in the Early Intermediate Period Ayacucho: a view from the site of Nawinpukyo, en: W. H. Isbell y H. Silverman (eds.), Andean Archaeology III: North and South, 279-306, Springer, New York. https://doi.org/10.1007/0-387-28940-2_13

Massey, S. A.

1991 Social and political leadership in the lower Ica Valley: Ocucaje Phases 8 and 9, en: A. Paul (ed.), Paracas art and architecture, 349-416, University of Iowa Press, Iowa City.

Miller, G. R. y R. L. Burger

1995 Our father the cayman, our dinner the llama: animal utilization at Chavin de Huantar, Peru, American Antiquity 60 (3), 421-458. https://doi.org/10.2307/282258

Murra, J. V.

1975 El control vertical de un máximo de pisos ecológicos en la economía de las sociedades andinas, en: J.V.

[1972] Murra, Formaciones económicas y politicas del mundo andino, 59-116, Instituto de Estudios Peruanos, Lima.

Oberem, U.

1976 El acceso a recursos naturales de diferentes ecologías en la sierra ecuatoriana (siglo XVI), ponencia presentada en el 42, Congrés International des Américanistes, París.

\section{Ochatoma Paravicino, J.}

1998 El Período Formativo en Ayacucho: balance y perspectivas, en: P. Kaulicke (ed.), Perspectivas Regionales del Período Formativo en el Perú, Boletín de Arqueología PUCP 2, 289-302.

Perevolotsky, A.

1985 Los pobladores de los despoblados: goat herders in Piura, Peru, Small Ruminant Collaborative Research Support Program 33, Department of Rural Sociology University of Missouri-Colombia/Division of Environmental Studies University of California-Davis, Columbia. 
Proulx, D. A.

2008 Paracas and Nasca: regional cultures on the South Coast of Peru, en: H. Silverman y W. H. Isbell (eds.), Handbook of South American Archaeology, 563-586, Springer, New York. https://doi.org/10.1007/978-0387-74907-5_29

Reindel, M.

2012 Berichte für die Jahre 2009-2010 der Projekte der Kommission für Archäologie Außereuropäischer Kulturen des Deutschen Archäologischen Instituts, Zeitschrift für Archäologie Außereuropäischer Kulturen 4, 369-432.

Reindel, M., J. Isla, H. Otten, H. Gorbahn y J. von Schwerin

2013 Archäologische forschungen in Peru und Honduras, Zeitschrift für Archäologie Außereuropäischer Kulturen 5, 297-313.

Reindel, M., J. Isla, H. Gorbahn y H. Otten

2015 Paracas en Palpa: los fundamentos del poder de la cultura Nasca, Peruvian Archaeology 2 (La cultura Paracas en la costa sur del Perú), 37-64.

Renneberg, R., S. Hummel y B. Herrmann

2009 The Nasca and their dear creatures - molecular genetic analysis of pre-Columbian camelid bones and textiles, en: M. Reindel y G. A. Wagner, (eds.), New technologies for archaeology: multidisciplinary investigations in Palpa and Nasca, Peru, 193-203, Springer-Verlag, Berlín. https://doi.org/10.1007/978-3-54087438-6_12

Rowe, J. H.

1946 Inca culture at the time of the Spanish conquest, en: J. H. Steward (ed.), Handbook of South American Indians, 183-330, vol. 2: The Andean Civilization. Smithsonian Institution, Bureau of American Ethnography, Bulletin No. 143, Washington, D.C.

Schreiber, Katharina J.

2000 Los wari en su contexto local: Nasca y Sondondo, en: P. Kaulicke y W. H. Isbell, (eds.), Huari y Tiwanaku: Modelos vs. Evidencias. Primera Parte, Boletin de Arqueología PUCP 4, 425-448.

Schwartz, G. M. y J. J. Nichols (eds.)

2006 After Collapse: The Regeneration of Complex Societies, University of Arizona Press, Tucson.

Shimada, I.

1985 Introduction, en: S. Masuda, I. Shimada y C. Morris (eds.), Andean ecology and civilization: an interdisciplinary perspective on Andean ecological complementarity, xi-xxxii, University of Tokyo Press, Tokyo.

Silverman, $\mathrm{H}$.

2002 Ancient Nasca settlement and society, University of Iowa Press, Iowa City.

Silverman, H. y D. A. Proulx

2002 The Nasca, Blackwell Publishing, Malden.

Sossna, V.

2016 Climate and settlement in southern Peru: the Northern Rio Grande de Nasca Drainage Between 1500 $B C E$ and 1532 CE, Forschungen Zur Archaologie Aussereuropaischer Kulturen 13, Reichert Verlag, Wiesbaden.

Whalen, V. H.

2014 Re-becoming Nasca: a household-based analysis of the transformation of community and tradition at a Late Nasca village, Peru, tesis de Doctorado, Purdue University, West Lafayette.

Whaley, O. Q., D. G. Beresford-Jones, W. Milliken, A. Orellana, A. Smyk y J. Leguía

2010 An ecosystem approach to restoration and sustainable management of dry forest in Southern Peru, Kew Bulletin 65, 613-641. 